\title{
25 Research Square \\ Hospital-acquired COVID-19: a New Dilemma for the Infection Control Practitioner
}

\section{Eduardo Perez-Alba}

Hospital Universitario Dr Jose Eleuterio Gonzalez

Laura Nuzzolo-Shihadeh

Hospital Universitario Dr Jose Eleuterio Gonzalez

Magaly Padilla-Orozco

Hospital Universitario Dr Jose eleuterio gonzalez

marisela Mendoza-Garza

Hospital Universitario Dr Jose Eleuterio gonzalez

Adrián Camacho-Ortiz ( $\square$ acamacho_md@yahoo.com )

Hospital Universitario Dr. José eleuterio González https://orcid.org/0000-0002-8044-0094

\section{Research Article}

Keywords: COVID-19, SARS-CoV-2, Hospital-acquired, Nosocomial, Community-acquired

Posted Date: December 18th, 2020

DOl: https://doi.org/10.21203/rs.3.rs-129923/v1

License: (c) (1) This work is licensed under a Creative Commons Attribution 4.0 International License. Read Full License 


\section{Abstract}

\section{Background}

Preliminary reports show that nosocomial SARS-CoV-2 infection is not associated with increased mortality compared with community acquired infection.

\section{Methods}

Retrospective comparison of COVID-19 adult patients who were classified according to probable time of acquisition of SARS-CoV-2 and symptom onset. Data from hospitalized patients that were hospitalized in non-COVID-19 areas were reviewed.

All patients were classified as community-acquired/Community-onset (CA-CO), Communityacquired/hospital-onset (CA-HO) and Hospital-acquired/Hospital-onset (HA-HO) cases. All patients without respiratory symptoms were tested on day one and if negative, hospitalized in non-COVID-19 areas.

\section{Results}

We identified 59 patients that fulfilled the definition of CA-HO or HA-HO COVID-19. Patients in the CA-CO group were less likely to have multiple comorbidities than the patients in the CA-HO and HA-HO groups. Mortality was lower in the CA-CO group (21.8\%) compared to the other groups, although it did not reach statistical significance.

\section{Discussion}

We identified 9 clusters of HA-HO cases arising from multiple-bed rooms from the non-COVID-19 areas. There was no significant difference for HA-HO COVID-19 between patients placed in a common-room bed compared to patients placed on single bed rooms $(p=.19)$. Nevertheless, the RR for HA-HO COVID-19 was 105 (95\% Cl 62.9 to 177.6) for patients treated in a common-room allocating another COVID-19-detected patient within the immediate $24 \mathrm{~h}$ time frame $(\mathrm{P}=<0.01)$.

\section{Conclusion}

Hospital-acquired COVID-19 is newly described and poses a challenge for infection control. We identified small clusters related to multiple-bed rooms from non-COVID-19 hospitalization wards and propose a simple time-based classification for hospital surveillance and isolation precautions.

\section{Background}

The Coronavirus disease 2019 (COVID-19) pandemic has changed the way we perceive and treat patients outside and inside the hospital. ${ }^{1}$ The majority of patients who develop symptoms begin as outpatients and are commonly associated to community exposure. As hospitals increase their workload, strategies 
for pre-hospitalization triage may fall short due to false negative results on qPCR testing, mild symptoms or asymptomatic or pre-symptomatic infection. ${ }^{2}$

While classification of COVID-19 has focused on severity, radiologic markers, prognostic features, and serologic markers; there is a lack of an epidemiologic classification. ${ }^{3-5}$ Preliminary data showed that nosocomial severe acute respiratory syndrome coronavirus 2 (SARS-CoV-2) infection was not associated with increased mortality when compared to community-acquired infection. ${ }^{6}$ Rhee et al. reported a low incidence of nosocomial SARS-CoV-2 infection rate using an intricate classification employing the terms "likely" and "definitively" for community-acquired and hospital-acquired cases and found a very low incidence of COVID-19 definitely acquired in the hospital setting ${ }^{7}$.

Our understanding of the incubation period plays a fundamental part in establishing where the patient contracted the viral infection and where the patient developed symptoms. The median incubation period for COVID-19 is estimated to be $5-5.1$ days ( $95 \% \mathrm{Cl}, 4.5$ to 5.8 days), furthermore $97.5 \%$ of the patients who develop symptoms will do before 11.5 days (Cl, 8.2 to 15.6 days). ${ }^{8-9}$ We classified hospitalized cases of COVID-19 based on calculated time of acquisition and incubation period of SARS-CoV-2 and present our findings.

\section{Material And Methods}

\section{Ethics approval}

The study was approved by the local ethics committee with the number IF20-00026.

\section{Study Design}

Retrospective comparison of adult patients with COVID-19 diagnosis who were classified according to probable time of acquisition of SARS-CoV-2 and symptom onset. Data from hospitalized patients were reviewed from June 1 to September 30, 2020.

\section{Setting}

The university hospital is a multi-building complex that includes two separate hospitals and many different buildings for diagnosis and treatment of multiple diseases. The main building is a 600-bed hospital designated for the treatment of non-COVID-19 cases; It has a 20-bed adult ICU, a 20-bed neonatal/pediatric ICU and various medical and surgical wards composed of multiple bed commonrooms (from 2 to 10 bed-rooms) for patient care with few individual rooms. Common-bed rooms do not have negative pressure systems and have common air conditioning ducts. Hospitalization characteristics regarding patient placement in common and single room were registered.

The second building is a 76-bed hospital designated for the diagnosis and treatment of suspected or confirmed COVID-19 cases. All patients with respiratory symptoms compatible with COVID-19 are directed to this hospital by a pre-arrival or pre-hospital triage. All patients that were hospitalized by other causes in 
the non-COVID-19 areas that subsequently developed COVID-19 or with a new detectable qPCR test for SARS-CoV-2 were transferred to the COVID-19 hospital at any point during care.

Patients from the COVID-19 hospital who were deemed non-transmissible (14 days after symptom onset and afebrile for at least $72 \mathrm{hrs}$ ) could be transferred to the main building for treatment continuity (nonCOVID-19) after the infection control team authorized it.

\section{Testing}

After May 30th 2020, all patients without suspected COVID-19 that required hospitalization were isolated in a specific hospital ward in individual rooms and were tested for SARS-CoV-2 by nasopharyngeal swab during their first $24 \mathrm{~h}$ of hospital stay. After a non-detectable result was obtained, they were hospitalized in the corresponding treatments wards.

At any time during hospitalization if a patient developed fever and/or signs and symptoms compatible with COVID-19 they were re-tested and if detectable they were transferred to the COVID-19 hospital. If a patient was hospitalized in a multiple-bed room then all the patients in the room were swabbed within $24 \mathrm{~h}$ and screened for symptoms.

All patients with suspected SARS-CoV-2 infection were cared for in the COVID-19 hospital and were swabbed upon admission and a second swab was ordered if the first one was non-detectable.

\section{Classification and definitions}

Community-acquired/Community-onset (CA-CO): for patients that had a positive qPCR test and developed COVID-19 symptoms before arriving to the hospital and had not been hospitalized or had not visited any healthcare facility in the two previous weeks.

Community-acquired/Hospital-onset (CA-HO): for patients that had a negative qPCR test and had no COVID-19 related symptoms upon admission to the hospital, and subsequently had a positive qPCR test with or without symptoms of COVID-19 during the first six days after hospitalization. These patients had not been hospitalized or had not visited any healthcare facility in the two previous weeks.

Hospital-acquired/Hospital-onset (HA-HO): for patients that had negative qPCR test and had no COVID-19 related symptoms since hospital admission or until day six of hospitalization, and subsequently had a positive qPCR test with or without symptoms of COVID-19 seven or more days after admission. These patients had not been hospitalized or in any healthcare facility in the two previous weeks.

Hospital-acquired/Community-onset (HA-CO): for patients who developed COVID-19 related symptoms and or had a positive qPCR test during the first 6 days after hospital discharge (Fig. 1). Follow-up in this group was limited and unreliable thus we decided not to include it in the analysis.

\section{RT-PCR testing for SARS-CoV-2}

We used real time qPCR testing using ThermoFisher@ and the Berlin protocol as previously described ${ }^{10}$. 


\section{Statistical analysis}

We used dispersion classification for demographic data using mean and interquartile measurements. For dichotomous variables Chi square and Fishers exact test were used and for continuous variables Wilcoxon test was employed. ANOVA was used for 3 group comparison. A p value of $<.05$ was considered as statistically significant. MedCalc and SPSS ver. 20 were used.

\section{Results}

During the study period we evaluated 6967 total patients in our institution. Of those, 658 had respiratory symptoms compatible with COVID-19 and 6309 patients did not have respiratory symptoms upon initial evaluation. A total of 492 patients were hospitalized directly in our COVID-19 area, all other patients that required hospitalization for non-COVID-19 conditions were screened in day one. The latter strategy rendered 166 detections and transfers to the COVID-19 area without being hospitalized in a non-COVID-19 area.

Out of all the hospitalized patients in non-COVID-19 areas who were negative on initial qPCR-test and had no respiratory symptoms upon admission, $59(0.93 \%)$ subsequently had a detectable test (10 between day two and six after hospitalization and 49 at day $\geq 7$ after hospitalization). There were no differences between the groups regarding gender or age. Patients in the CA-CO group were less likely to have malignancy, chronic kidney disease and had a lower comorbidity index than the patients in the CA-HO and HA-HO groups (Table 1). 
Table 1

Clinical aspects and patient characteristics of the different COVID-19 patients by the time of symptom onset.

\begin{tabular}{|c|c|c|c|c|c|}
\hline Characteristic & $\begin{array}{l}\text { Total } n= \\
650\end{array}$ & $\begin{array}{l}\text { CA-CO n = } \\
591\end{array}$ & $\begin{array}{l}\text { CA-HO } \\
n=10\end{array}$ & $\begin{array}{l}\mathrm{HA}-\mathrm{HO} n= \\
49\end{array}$ & $\mathrm{p}$ \\
\hline Male gender & $401(61.69 \%)$ & $368(62.26 \%)$ & $5(50 \%)$ & $28(57.14 \%)$ & .57 \\
\hline Age & $\begin{array}{l}54.7( \pm \\
16.73)\end{array}$ & $\begin{array}{l}55.13( \pm \\
16.5)\end{array}$ & $\begin{array}{l}54.9( \pm \\
16.06)\end{array}$ & $\begin{array}{l}50.34( \pm \\
19.13)\end{array}$ & .15 \\
\hline \multicolumn{6}{|l|}{ Comorbidity } \\
\hline Obesity & $77(11.84 \%)$ & $74(12.52 \%)$ & $2(20 \%)$ & $1(2 \%)$ & .06 \\
\hline Diabetes & $260(40 \%)$ & $236(39.93 \%)$ & $7(70 \%)$ & $17(34.69 \%)$ & .11 \\
\hline Hypertension & $\begin{array}{l}215 \\
(33.07 \%)\end{array}$ & 195(32.99\%) & $4(40 \%)$ & $16(32.65 \%)$ & .89 \\
\hline Chronic kidney disease & $53(8.15 \%)$ & $41(6.93 \%)$ & $1(10 \%)$ & $11(22.44 \%)$ & $<.01$ \\
\hline Chronic heart disease & $42(6.46 \%)$ & $41(6.93 \%)$ & $1(10 \%)$ & $0(0 \%)$ & .70 \\
\hline Malignancy & $45(6.9 \%)$ & $34(5.75 \%)$ & $0(0 \%)$ & $11(22.44 \%)$ & $<.01$ \\
\hline Smoking history & $29(4.46 \%)$ & $25(4.23 \%)$ & $0(0 \%)$ & $4(8.16 \%)$ & .20 \\
\hline Charlson comorbidity index & $2.41( \pm 1.94)$ & $2.3( \pm 1.91)$ & $4( \pm 1.63)$ & $\begin{array}{l}3.06( \pm \\
2.08)\end{array}$ & $<.01$ \\
\hline \multicolumn{6}{|l|}{ Oxygenation requirements } \\
\hline NEWS score & $5( \pm 3.18)$ & $5.06( \pm 3.14)$ & $\begin{array}{l}5.8( \pm \\
2.74)\end{array}$ & $4.9( \pm 3.77)$ & 0.73 \\
\hline $\begin{array}{l}\text { initial need for oxygen } \\
\text { supplementation }\end{array}$ & $444(68.3 \%)$ & $415(70.21 \%)$ & $6(60 \%)$ & $23(46.93 \%)$ & $<.01$ \\
\hline High-flow nasal canula & $183(28.15 \%)$ & $176(29.78 \%)$ & $1(10 \%)$ & $6(12.24 \%)$ & .01 \\
\hline Invasive mechanical ventilation & $168(25.84 \%)$ & $153(25.88 \%)$ & $2(20 \%)$ & $13(26.53 \%)$ & .90 \\
\hline Renal replacement therapy & $83(12.76 \%)$ & $67(11.33 \%)$ & $3(30 \%)$ & $13(26.53 \%)$ & $<.01$ \\
\hline Days from symptom onset & $6.4( \pm 5.4)$ & $6.7( \pm 5.42)$ & $\begin{array}{l}7.1( \pm \\
5.83)\end{array}$ & $2.4( \pm 4.63)$ & .99 \\
\hline \multicolumn{6}{|l|}{ Outcomes } \\
\hline Length of hospital stay & $8.6( \pm 7.6)$ & $8.7( \pm 7.78)$ & $\begin{array}{l}4.9( \pm \\
3.87)\end{array}$ & $8.6( \pm 6.24)$ & .29 \\
\hline 30-day mortality & $149(22.92 \%)$ & $129(21.82 \%)$ & $4(40 \%)$ & $16(32.65 \%)$ & .09 \\
\hline
\end{tabular}




\begin{tabular}{|lllll|}
\hline Characteristic & $\begin{array}{l}\text { Total } n= \\
650\end{array}$ & $\begin{array}{l}\text { CA-CO } n= \\
591\end{array}$ & $\begin{array}{c}\text { CA-HO } \\
n=10\end{array}$ & $\begin{array}{l}\text { HA-HO } n=~ \\
49\end{array}$ \\
\hline CA-HO, Community-acquired / hospital onset & & \\
\hline HA-HO, Hospital-acquired/ hospital onset & & \\
\hline
\end{tabular}

Patients from the HA-HO group were less likely to require any type of oxygen supplementation upon diagnosis and less likely to require High-Flow Oxygenation $\left(\mathrm{HFO}_{2}\right)$ at any point during hospitalization, although they had a statistically higher proportion of renal replacement therapy.

Overall mortality was lower in the CA-CO group $(21.8 \%)$ compared to the CA-HO (40.0\%) and the HA-HO $(32.1 \%)$ groups, although it did not reach statistical significance. We identified nine small clusters of HA$\mathrm{HO}$ cases arising from multiple-bed rooms from the non-COVID-19 areas: six pairs of infected patients from six-bed rooms, a couple of four patient clusters from eight-bed rooms and a five patient cluster from a ten-bed room.

There was no significant difference for HA-HO COVID-19 between the patients placed in a common-room bed (47 of 6309 patients) compared to patients placed on single-bed rooms (two of 103) $(p=.19)$. We found that the RR for HA-HO COVID-19 was 105 (95\% Cl 62.9 to 177.6) for patients treated in a commonroom that had another COVID-19 detected patient within the immediate $24 \mathrm{~h}$ time frame $(P=<.01)$. Follow-up data for HA-CO patients was not reliable for analysis and was not included.

\section{Discussion}

An overwhelming proportion of pre-symptomatic patients are highly contagious and, at that point in the natural history of the disease, diagnostic testing is sub-optimal. These factors pose an important challenge for infection control in hospitals and medical facilities.

Nosocomial acquisition of SARS-CoV-2 can vary widely from $1.7-44 \%$; we report $0.77 \%$ in the present study based in our classification. ${ }^{7,11}$ Multiple factors influence these proportions such as personal protective equipment, mandatory masking and visitor restrictions, ${ }^{14}$ among others. Although in our series multiple-bed rooms probably played a negative role for in-hospital dissemination of SARS-CoV-2 since $44.8 \%$ of Hospital acquired/Hospital onset cases shared a room with at least one breakthrough positive case. $^{12,13}$

This poses a great challenge for hospitals with multiple occupancy rooms and lack of negative pressure rooms in pandemic settings for COVID-19 patient care. The classification is also based on the availability of a qPCR universal screening of patients upon hospital admission most of whom did not have respiratory symptoms. 
Classifying patients with traditionally community-acquired infections in the healthcare setting is a complex task. Hospital-acquired influenza for instance has shown different clinical characteristics and outcomes, and cases often occurred in clusters making intra-hospital transmission highly probable. ${ }^{15,16}$ This highlights the importance of infection control measures to limit the spread of influenza and other virus such as SARS-Cov-2. We identified small clusters of patients in multiple-bed rooms that were epidemiologically linked. During the study period visitation was restricted to one person per patient in the ICU, but visits were not allowed in areas where the clusters occurred. Thus, patients with long incubation periods and infected healthcare personnel were the probable causes of breakthrough cases.

We identified limitations to our study. First, in the absence of whole genome sequencing or next generation sequencing the stated classification might misclassify some cases. Based on Lauer SA et al, $5 \%$ of SARS-CoV-2 infected patients have a greater or shorter incubation period. Thus, some patients classified as CA-HO might be in fact purely community related and a similar case could be made for HA$\mathrm{CO}$ after the sixth day of discharge. ${ }^{9}$ Also, false negative results for qPCR-based testing might contribute to misclassification of some patients. Follow-up of discharged patients for HA-CO cases was not entirely performed.

\section{Conclusion}

Hospital acquired COVID-19 is a newly described entity and poses a challenge for infection control. We identified small clusters related to multiple-bed rooms from non-COVID-19 hospitalization wards and propose a simple time-based classification that will improve hospital surveillance and isolation precautions.

\section{Declarations}

- Ethical Approval and Consent to participate: Local ethics committee IF20-00026.

- Consent for publication: not applicable

- Availability of supporting data: yes

- Competing interests: None to declare

- Funding: No funding

- Authors' contributions: EPA, LNS gathered the data, reviewed the final manuscript, MPO designed the study, MMG gathered the data, ACO designed the study, gathered the data, wrote the initial manuscript and reviewed the final manuscript

- Acknowledgements: We acknowledge the help from the infection control unit from the university hospital for their tremendous work ethic

\section{References}


1. Ge H, Wang X, Yuan X, Xiao G, Wang C, Deng T, Yuan Q, Xiao X. The epidemiology and clinical information about COVID-19. Eur J Clin Microbiol Infect Dis. 2020 Jun;39(6):1011-1019. doi: 10.1007/s1 0096-020-03874-z. Epub 2020 Apr 14. PMID: 32291542; PMCID: PMC7154215.

2. Kucirka LM, Lauer SA, Laeyendecker O, Boon D, Lessler J. Variation in False-Negative Rate of Reverse Transcriptase Polymerase Chain Reaction-Based SARS-CoV-2 Tests by Time Since Exposure. Ann Intern Med. 2020 Aug 18;173(4):262-267. doi: 10.7326/M20-1495. Epub 2020 May 13. PMID: 32422057; PMCID: PMC7240870.

3. Clinical Management of COVID-19 interim guidance. WHO REFERENCE NUMBER: WHO/2019nCoV/clinical/2020.5 at https://www.who.int/publications/i/item/clinical-management-of-covid-19 (last accessed October 19, 2020)

4. Li K, Wu J, Wu F, Guo D, Chen L, Fang Z, Li C. The Clinical and Chest CT Features Associated With Severe and Critical COVID-19 Pneumonia. Invest Radiol. 2020 Jun;55(6):327-331. doi: 10.1097/RLI.0000000000000672. PMID: 32118615; PMCID: PMC7147273.

5. Li X, Xu S, Yu M, Wang K, Tao Y, Zhou Y, Shi J, Zhou M, Wu B, Yang Z, Zhang C, Yue J, Zhang Z, Renz H, Liu X, Xie J, Xie M, Zhao J. Risk factors for severity and mortality in adult COVID-19 inpatients in Wuhan. J Allergy Clin Immunol. 2020 Jul;146(1):110-118. doi: 10.1016/j.jaci.2020.04.006. Epub 2020 Apr 12. PMID: 32294485; PMCID: PMC7152876.

6. Khan KS, Reed-Embleton H, Lewis J, Saldanha J, Mahmud S. Does nosocomial SARS-CoV-2 infection result in increased 30-day mortality? A multi-centre observational study to identify risk factors for worse outcomes in COVID-19 disease. J Hosp Infect. 2020 Sep 17:S0195-6701(20)30432-1. doi: 10.1016/j.jhin.2020.09.017. Epub ahead of print. PMID: 32950587; PMCID: PMC7495174.

7. Rhee C, Baker M, Vaidya V, Tucker R, Resnick A, Morris CA, Klompas M; CDC Prevention Epicenters Program. Incidence of Nosocomial COVID-19 in Patients Hospitalized at a Large US Academic Medical Center. JAMA Netw Open. 2020 Sep 1;3(9):e2020498. doi: 10.1001/jamanetworkopen.2020.20498. PMID: 32902653; PMCID: PMC7489854.

8. Lauer SA, Grantz KH, Bi Q, Jones FK, Zheng Q, Meredith HR, Azman AS, Reich NG, Lessler J. The Incubation Period of Coronavirus Disease 2019 (COVID-19) From Publicly Reported Confirmed Cases: Estimation and Application. Ann Intern Med. 2020 May 5;172(9):577-582. doi: 10.7326/M200504. Epub 2020 Mar 10. PMID: 32150748; PMCID: PMC7081172.

9. Nie X, Fan L, Mu G, Tan Q, Wang M, Xie Y, Cao L, Zhou M, Zhang Z, Chen W. Epidemiological Characteristics and Incubation Period of 7015 Confirmed Cases With Coronavirus Disease 2019 Outside Hubei Province in China. J Infect Dis. 2020 Jun 16;222(1):26-33. doi: 10.1093/infdis/jiaa211. PMID: 32339231; PMCID: PMC7197553.

10. https://www.who.int/docs/default-source/coronaviruse/wuhan-virus-assayv1991527e5122341d99287a1b17c111902.pdf (last accessed November 15, 2020).

11. Zhou Q, Gao Y, Wang X, et al; COVID-19 Evidence and Recommendations Working Group. Nosocomial infections among patients with COVID-19, SARS and MERS: a rapid review and metaanalysis. Ann Transl Med. 2020;8(10):629. doi:10.21037/atm-20-3324 
12. Houghton C, Meskell P, Delaney H, Smalle M, Glenton C, Booth A, Chan XHS, Devane D, Biesty LM. Barriers and facilitators to healthcare workers' adherence with infection prevention and control (IPC) guidelines for respiratory infectious diseases: a rapid qualitative evidence synthesis. Cochrane Database Syst Rev. 2020 Apr 21;4(4):CD013582. doi: 10.1002/14651858.CD013582. PMID: 32315451 ; PMCID: PMC7173761.

13. Kitano T, Piché-Renaud PP, Groves HE, Streitenberger L, Freeman R, Science M. Visitor Restriction Policy on Pediatric Wards during Novel Coronavirus (COVID-19) Outbreak: A Survey Study across North America. J Pediatric Infect Dis Soc. 2020 Oct 22:piaa126. doi: 10.1093/jpids/piaa126. Epub ahead of print. PMID: 33090211.

14. Álvarez-Lerma F, Marín-Corral J, Vilà C, Masclans JR, Loeches IM, Barbadillo S, González de Molina FJ, Rodríguez A; H1N1 GETGAG/SEMICYUC Study Group. Characteristics of patients with hospitalacquired influenza A (H1N1)pdm09 virus admitted to the intensive care unit. J Hosp Infect. 2017 Feb;95(2):200-206. doi: 10.1016/j.jhin.2016.12.017. Epub 2016 Dec 30. PMID: 28153560.

15. Parkash N, Beckingham W, Andersson P, Kelly P, Senanayake S, Coatsworth N. Hospital-acquired influenza in an Australian tertiary Centre 2017: a surveillance based study. BMC Pulm Med. 2019 Apr 16;19(1):79. doi: 10.1186/s12890-019-0842-6. PMID: 30991976; PMCID: PMC6469028.

\section{Figures}

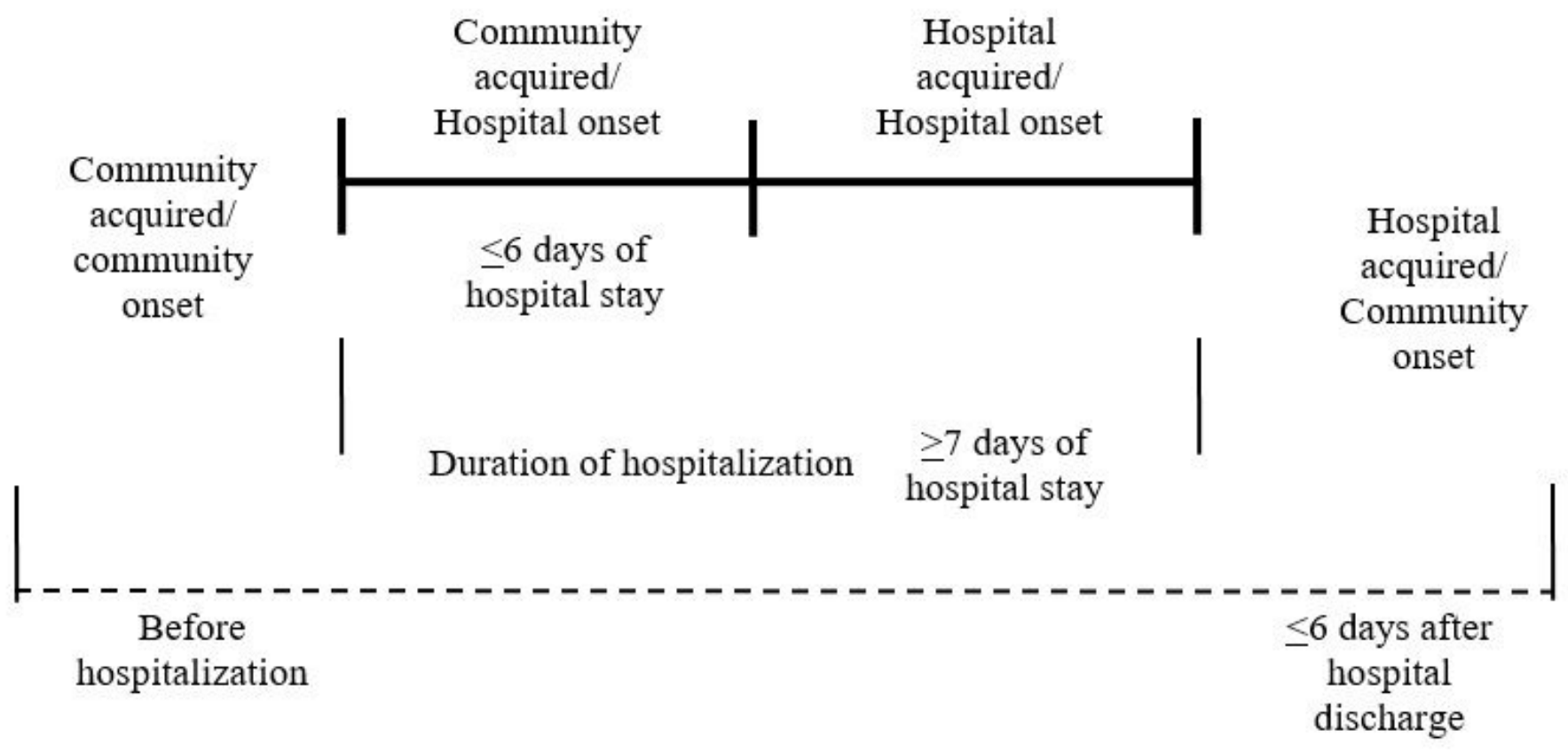

Figure 1 
Schematics used for classification of COVID-19 cases and their epidemiologic relation to acquisition and disease onset 